\title{
Research on the $k$-Coverage Local Wireless Network and Its Communication Coordination Mechanism Design
}

\author{
Rongchang Yuan ${ }^{1,2}$, Haigan Yuan $^{3}$, Si Chen ${ }^{4}$, Longqing Sun ${ }^{2, *}$, Feng Qin ${ }^{2}$, \\ Han Zhang ${ }^{5}$,Yukun $\mathrm{Zhu}^{2}$, and Daokun $\mathrm{Ma}^{2}$ \\ ${ }^{1}$ Power Automation Department, China Electric Power Research Institute, \\ Beijing100192, P.R. China \\ ${ }^{2}$ College of Information and Electrical Engineering, China Agricultural University, \\ Beijing 100083, P.R. China \\ ${ }^{3}$ School of sciences, South China University of Technology, GuangZhou510640, P.R. China \\ ${ }^{4}$ Department of Electrical Engineering, \\ State University of New York (SUNY) at Buffalo, New York 14260, U.S. \\ ${ }^{5}$ School of management, Tianjin University, Tianjin300072, P.R. China \\ sunlq@eau . edu.cn
}

\begin{abstract}
Based on the analysis of the present situation and features of local wireless network, combined the networking technologies, we constructing the wireless network layout algorithm and sensor nodes model. Through delaminating iterative optimization algorithm, optimal layout of sensor data collection and satisfy recognition, quasi-geoids orientation, signal coverage optimization have been realized. Compared the triangular, square and hexagon scheme, and get a general scheme of multiple complete coverage by calculate the length of sides on the polygon. Monte Carlo algorithm is used in choosing the optimum proposal comparing among the regular triangle, square and the hexagon in different conditions. For locating network, analyze the advantages and disadvantages of triangle, square and hexagon schemes in deferent positioning accuracy of double coverage, we set different layout schemes to different situations. Application manual is provided for the constructor to refer to these table lookup to attain the right numerical value.
\end{abstract}

Keywords: $k$-coverage, local wireless network, communication coordination, Monte Carlo.

\section{Introduction}

In the subject of internet of things, different kinds of information are transferred by sensor nodes. In order to improve the efficiency and stability of the transmission through networks, the layout of the nodes must be optimized. (Zhang Honghai and Hou Jennifer,2005; Yu Hongyi,2008).

Zigbee is one of the hardware equipment used in the wireless communication technology. It has many advantages, for example, the connection distance is shorter

\footnotetext{
Corresponding author.
} 
and the complexity is smaller, the power consumption is lower and so on. In the automatic control and remote control field, it is widespread. Many communicators are inset with Zigbee. It consists of three models: the response model; the data manipulation model; the communication model. The response model is to receive the information. The information is transferred through the communication model. With such information, we can attain the location of the moving nodes. (Duc A. Tran, Usman Khan, 2006)

\section{Methods and Algorithms}

\subsection{The Network Evaluation Function}

When to evaluate a network, we often consider the following two factors: the network performance and the cost. The network performance includes the stability in transfer information and the accuracy in location. It depends on the overlapping area. The cost depends on the number of nodes. The more nodes there are the higher cost and power consumption.

When the target area is $k$-coverage, the size of area covered is:

$$
S_{\text {becovered }}=a b
$$

If the number of nodes in this area is $n$, the coverage area is:

$$
S_{\text {all }}=n \pi R^{2}
$$

Where: $a$ is the length of the target area,

$b$ is the width of the target area

$R$ is the effective communication radius.

At the same time, every point in the target area has the very same information collection accuracy.

Definition 1. The coverage rate of sensor $\eta$

This passage comes up with the concept of coverage rate. It means the ratio between the size of target area and the sum of all the coverage area provided by the nodes.

$$
\eta=\frac{\bigcup_{i=1}^{n} S_{i}}{\sum_{i=1}^{n} S_{i}}
$$

It can reflect on the utilization of the coverage area. The larger $\eta$, the better the net performance is. At the same time, it can reflect the number of nodes. The fewer the nodes are, the lower the cost is. In another words, the lower the coverage rate, the better the layout of the network. 
In a 1-coverage network, the calculation function is defined:

$$
g_{1}=\left(S_{\text {cover }}-S_{\text {becovered }}\right) / S_{\text {becovered }}=\frac{1}{\eta}-1
$$

Through the discussion, the coverage rate is solved. In the 1-coverage network, it is $82.7 \%$. In fact, it can't reach the perfect value 1 .

In a $k$-coverage network, the covered area can be considered as:

$$
k S_{\text {becovered }}=k a b
$$

All in all, in a $k$-coverage network, the calculation function is defined:

$$
g_{k}=\left(S_{\text {cover }}-S_{\text {becovered }}\right) / S_{\text {becovered }}=\frac{k}{\eta}-1
$$

\subsection{The Layout of Nodes in 1-Coverage Network}

To design the nodes layout, for a target area, the main problem is how to make the area is covered without blind spot, which means that the point can't be covered. According to the simplification of the covered area-the circle, we can turn the problem into geometric. For a $a b$ rectangle region, how to be fitted together can use the least circles and how many circles are needed. As no matter small the radius of the circle is, it impossible to cover the area without blind point, so we take the second best solution that the circle is replaced with its inscribed polygon and the node is located on the center of the polygon.

If there are $x$ polygons put on the same vertex, and every $n$ gon's interior angle is:

$$
(n-2) * 180^{\circ} / n
$$

Then:

$$
x \frac{(n-2) * 180^{\circ}}{n}=360^{\circ}
$$

The solution is:

$$
x=2+\frac{4}{n-2}
$$

Because $x$ is positive integer, then:

$$
\begin{aligned}
& n=3, x_{3}=6 ; \\
& n=4, x_{4}=4 ; \\
& n=6, x_{6}=3 ;
\end{aligned}
$$


From that we can see the polygon can be regular triangle, square and the hexagon like the following Figure 1 shows.

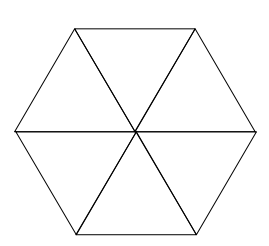

(a)

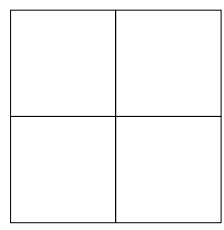

(b)

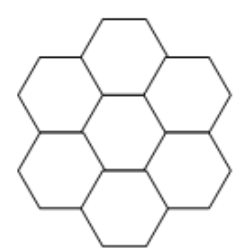

(c)

Fig. 1. Three different kinds of repetitive units

Locate the nodes according to the above way, then we can get the covered area without blind points.

$$
A_{C}=A_{S}-A_{L}=\pi R^{2}-\frac{n R^{2}}{2} \sin \frac{2 \pi}{n}
$$

Where:

$A_{C}$ means the size of overlapping area.

$A_{L}$ means the size of the circle with the effective communication radius.

$A_{S}$ means the size of inscribed polygon. $n$ is the number of the sides on the polygon.

When $n \rightarrow \infty, A_{C} \rightarrow 0$. So in order to make sure, there is no blind node. When $n=6$, the overlapping area is minimum:

$$
A_{C \min }=\left(\pi-\frac{3 \sqrt{2}}{2}\right) R^{2}
$$

Using the hexagon to locate the nodes is the best layout(Zhang Chaohui,2010).

Using the Definition 1 in the model one, we can calculate the coverage rate $\eta_{S}$ :

$$
\eta_{S}=\frac{A_{S}}{A_{L}}=\frac{3 \sqrt{3} R^{2} / 2}{\pi R^{2}}=82.7 \%
$$

Then the calculation function is:

$$
g_{1}=\frac{1}{\eta_{S}}-1=0.2092
$$




\subsection{The Layout of Nodes in $k$-Coverage Network}

During the research on $k$-coverage network, the two puzzles challenge us. One is that if the geometric shapes in $k$-coverage network is the same to the 1 -coverage network. Another one is that how to calculate the distance between the two nodes.

We regard the second question as the breakthrough point to compute the distance between two nodes in $k$-coverage network. we have proven that there are three possibilities: regular triangle, square and the hexagon. And the node is located on the vertex.

We can see the vertex is the most difficult point to be covered. So if the vertex is covered by $k$ times, the area is covered by $k$ times.

We select one of the vertex $M$, to solve $\left(x_{M}, y_{M}\right)$ using the equation of the straight lines: $L_{1}, L_{2}$. In order to make sure $M$ is covered $k$ times, which is that there are $\mathrm{k}$ nodes lie in the effective communication circle of $M$ :

$$
\sqrt{\left(x_{M}-x_{N}\right)^{2}+\left(y_{M}-y_{N}\right)^{2}} \leq R^{2}
$$

$\left(x_{N}, y_{N}\right)$ is one of nodes $N$ surrounded the node $M$.

Define a distance function:

$$
G=R^{2}-\sqrt{\left(x_{M}-x_{N}\right)^{2}+\left(y_{M}-y_{N}\right)^{2}}
$$

Only if $\mathrm{G}>0$, the target area is $k$-coverage.

Assume that: $\mathrm{N}$ is the point of intersection between $L_{1}{ }^{\prime}, L_{2}{ }^{\prime}$,

Then we can get the $L_{1}{ }^{\prime}, L_{2}{ }^{\prime}$ through translating $L_{1} 、 L_{2}$, passing $k_{1}$ and $k_{2}$ units.

Then $N\left(x_{N}, y_{N}\right)$ can be expressed by $M\left(x_{M}, y_{M}\right)$.

$$
\begin{aligned}
& x_{N}=x_{M}\left(k_{1}\right) ; \\
& y_{N}=y_{M}\left(k_{2}\right) ;
\end{aligned}
$$

Then the distance $G$ can be expressed by $k_{1} k_{2}$.

$$
G\left(k_{1}, k_{2}\right)=R^{2}-\sqrt{\left(x_{M}-x_{N}\right)^{2}+\left(y_{M}-y_{N}\right)^{2}}
$$

We must find out the $k_{1} k_{2}$, then we can get the distance between $M$ and $N$. 


\section{(1) Solution}

Taking the 2-coverage for example, introduce above model in detail.

We have proved there are three shapes; regular triangle, square and the hexagon showed in the following Figure 2:

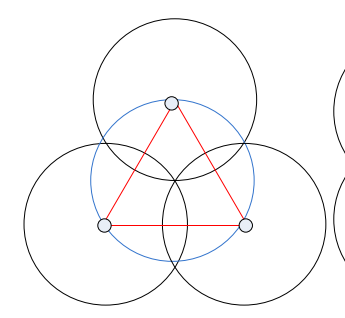

(a)

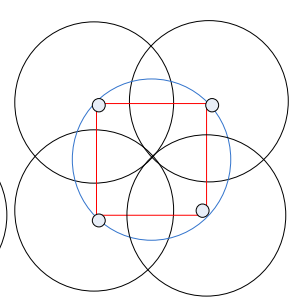

(d)

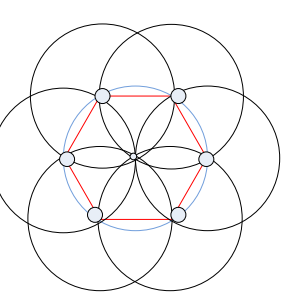

(c)

Fig. 2. The different shapes

From above Figure 2(a), we can see: when it is regular triangle, it has the least nodes

So we take the 2-coverage using regular triangle for example.

$L_{1}, L_{2}$ showed in the Figure 3:

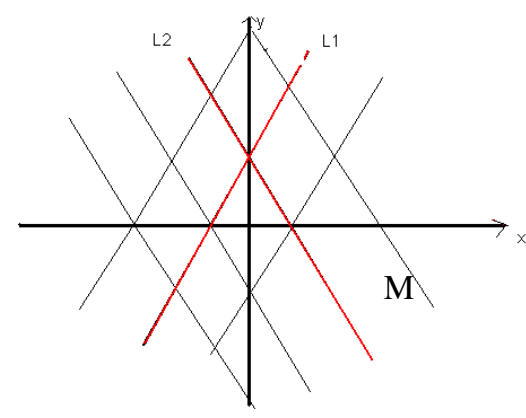

Fig. 3. 2-coverage using regular triangle

The equation of $L_{1}, L_{2}$ :

$$
\begin{aligned}
& y_{2}=\sqrt{3} x-\frac{\sqrt{3}}{2} m \\
& y_{2}=-\sqrt{3} x-\frac{\sqrt{3}}{2} m
\end{aligned}
$$

We select one of the vertexes $M(0, \sqrt{3} m / 2)$. 
Then we can get the $L_{1}{ }^{\prime}, L_{2}$ ' through translating $L_{1}, L_{2}$ passing $k_{1}$ and $k_{2}$ units

$$
\begin{gathered}
y_{1}=\sqrt{3}\left(x+k_{1} m\right)-m \sqrt{3} / 2 \\
y_{2}=-\sqrt{3}\left(x+k_{2} m\right)-m \sqrt{3} / 2
\end{gathered}
$$

Then coordinates of $N$ :

$$
\begin{gathered}
x_{N}=-\left(k_{1}+k_{2}\right) m / 2 \\
y_{N}=\left(k_{1}-k_{2}-1\right) \sqrt{3} m / 2
\end{gathered}
$$

Then the distances function:

$$
\begin{aligned}
& G=R_{2}-\left(x_{M}-x_{N}\right)^{2}+\left(y_{M}-y_{N}\right)^{2} \\
& =R_{2}-m_{2} / 4 *\left(\mathrm{k}_{1}+\mathrm{k}_{2}\right)^{2}+3 / 4 \mathrm{~m}^{2} *(\mathrm{k} 1-\mathrm{k} 2)^{2}
\end{aligned}
$$

For $k$-coverage, we can express $k$ using the multiply of $k_{1}$ and $k_{2}$.

It means the number of points of intersection.

$$
k=a b
$$

Where:

$$
k_{1}=a, k_{2}=b
$$

From that, we can get the numerical value. Then we can get the function: $G\left(k_{1}, k_{2}\right)$

$$
\begin{aligned}
& G=R^{2}-\left(x_{M}-x_{N}\right)^{2}+\left(y_{M}-y_{N}\right)^{2} \\
& =R^{2}-m^{2} / 4 *\left(k_{1}+k_{2}\right)^{2}+3 / 4 m^{2} *\left(k_{1}-k_{2}\right)^{2} \\
& =R^{2}-m^{2} / 4 *\left(k_{1}^{2}-k_{1} k_{2}+k_{2}^{2}\right)
\end{aligned}
$$

To simply the distance $G\left(k_{1}, k_{2}\right)$ into the $D\left(k_{1}, k_{2}\right)$ :

$$
D=k_{1}^{2}-k_{1} k_{2}+k_{2}^{2}
$$

Because $k_{1}$ and $k_{2}$ are all integer, we can get the value of them through gradual enlargement from $(0,0)$, for example: $(0,1),(0,-1),(-1,0) \ldots$... After getting the value of $D$, put the value in order of from small to bigger. The value order is the order to pick up $k_{1}, k_{2}$. 
We can get the value of the function $D$ showed in Table 1:

Table 1. The value of the function $D$ when $k_{1}, k_{2}$ change from -10 to 10

\begin{tabular}{|c|c|c|c|c|c|c|c|c|c|c|c|c|c|c|c|c|c|c|c|c|c|}
\hline & -10 & -9 & -8 & -7 & -6 & -5 & -4 & -3 & -2 & -1 & 0 & 1 & 2 & 3 & 4 & 5 & 6 & 7 & & 9 & 10 \\
\hline-10 & 100 & 91 & 84 & 79 & 76 & 75 & 76 & 79 & 84 & 91 & 100 & 111 & 124 & 139 & 156 & 175 & 196 & 219 & 244 & 271 & 300 \\
\hline-9 & 91 & 81 & 73 & 67 & 63 & 61 & 61 & 63 & 67 & 73 & 81 & 91 & 1031 & 117 & 133 & 151 & 171 & 193 & 217 & 243 & 271 \\
\hline-8 & 84 & 73 & 64 & 57 & 52 & 49 & 48 & 49 & 52 & 57 & 64 & 73 & 84 & 97 & 112 & 129 & 148 & 169 & 192 & 217 & 244 \\
\hline-7 & 79 & 67 & 57 & 49 & 43 & 39 & 37 & 37 & 39 & 43 & 49 & 57 & 67 & 79 & 93 & 109 & 127 & 147 & 169 & 193 & 219 \\
\hline-6 & 76 & 63 & 52 & 43 & 36 & 31 & 28 & 27 & 28 & 31 & 36 & 43 & 52 & 63 & 76 & 91 & 108 & 127 & 148 & 171 & 196 \\
\hline-5 & 75 & 61 & 49 & 39 & 31 & 25 & 21 & 19 & 19 & 21 & 25 & 31 & 39 & 49 & 61 & 75 & 91 & 109 & 129 & 151 & 175 \\
\hline-4 & 76 & 61 & 48 & 37 & 28 & 21 & 16 & 13 & 12 & 13 & 16 & 21 & 28 & 37 & 48 & 61 & 76 & 93 & 112 & 133 & 156 \\
\hline-3 & 79 & 63 & 49 & 37 & 27 & 19 & 13 & 9 & 7 & 7 & 9 & 13 & 19 & 27 & 37 & 49 & 63 & 79 & 97 & 117 & 139 \\
\hline-2 & 84 & 67 & 52 & 39 & 28 & 19 & 12 & 7 & 4 & 3 & 4 & 7 & 12 & 19 & 28 & 39 & 52 & 67 & 84 & 103 & 124 \\
\hline-1 & 91 & 73 & 57 & 43 & 31 & 21 & 13 & 7 & 3 & 1 & 1 & 3 & 7 & 13 & 21 & 31 & 43 & 57 & 73 & 91 & 111 \\
\hline 0 & 100 & 81 & 64 & 49 & 36 & 25 & 16 & 9 & 4 & & 0 & 1 & 4 & 9 & 16 & 25 & 36 & 49 & 64 & 81 & 100 \\
\hline 1 & 111 & 91 & 73 & 57 & 43 & 31 & 21 & 13 & 7 & & 1 & 1 & 3 & 7 & 13 & 21 & 31 & 43 & 57 & 73 & 91 \\
\hline 2 & 124 & 103 & 84 & 67 & 52 & 39 & 28 & 19 & 12 & 7 & 4 & 3 & 4 & 7 & 12 & 19 & 28 & 39 & 52 & 67 & 84 \\
\hline 3 & 139 & 117 & 97 & 79 & 63 & 49 & 37 & 27 & 19 & 13 & 9 & 7 & 7 & 9 & 13 & 19 & 27 & 37 & 49 & 63 & 79 \\
\hline 4 & 156 & 133 & 112 & 93 & 76 & 61 & 48 & 37 & 28 & 21 & 16 & 13 & 12 & 13 & 16 & 21 & 28 & 37 & 48 & 61 & 76 \\
\hline 5 & 175 & 151 & 129 & 109 & 91 & 75 & 61 & 49 & 39 & 31 & 25 & 21 & 19 & 19 & 21 & 25 & 31 & 39 & 49 & 61 & 75 \\
\hline 6 & 196 & 171 & 148 & 127 & 108 & 91 & 76 & 63 & 52 & 43 & 36 & 31 & 28 & 27 & 28 & 31 & 36 & 43 & 52 & 63 & 76 \\
\hline 7 & 19 & 193 & 169 & 147 & 127 & 109 & 93 & 79 & 67 & 5 & 49 & 43 & 39 & 37 & 37 & 39 & 43 & 49 & 57 & 67 & 79 \\
\hline 8 & 244 & 217 & 192 & 169 & 148 & 129 & 112 & 97 & 84 & 73 & 64 & 57 & 52 & 49 & 48 & 49 & 52 & 57 & 64 & 73 & 84 \\
\hline 9 & 271 & 243 & 217 & 193 & 171 & 151 & 1331 & 117 & 103 & 91 & 81 & 73 & 67 & 63 & 61 & 61 & 63 & 67 & 73 & 81 & 91 \\
\hline 10 & 300 & 271 & 244 & 2191 & 196 & 175 & 1561 & 139 & 124 & 111 & 100 & 91 & 84 & 79 & 76 & 75 & 76 & 79 & 84 & 91 & 100 \\
\hline
\end{tabular}

The constructor can refer to this application manual table lookup to attain the numerical value of $k_{1}$ and $k_{2}$, then they can get the best layout. Using the same solutions, we can get the results of square and the hexagon.

\section{(2) Algorithm}

The flow chart in $k$-coverage network, See Figure 4:

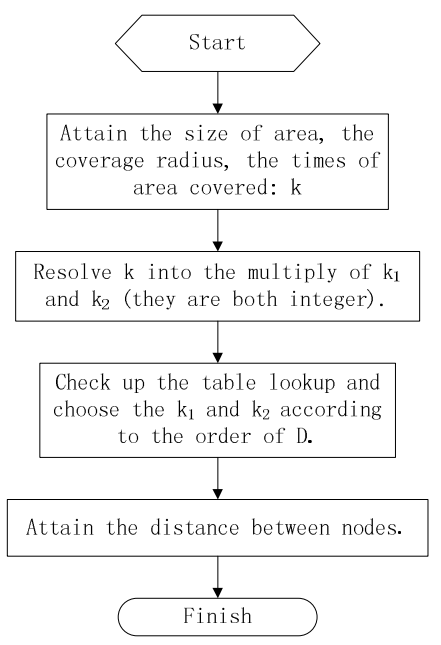

Fig. 4. Flow chart in k-coverage network

\section{(3) Cost of Network}

Definition 1. Minimum repeat unit: the minimum unit while attaching the nodes, namely, triangular, square and hexagonal. 
Definition 2. Node densities: the ratio of minimum repeat units and the number of the nodes in it. Symbol by $n$.

Because the cost of network only related to the numbers of nodes, the cost of a network can be expressed by $S / n$, where $S$ represent the area of minimum repeat unit.

Table 2. The value of $n$

\begin{tabular}{cc}
\hline scheme & $n$ \\
\hline triangular & $1 / 2$ \\
square & $3 / 4$ \\
hexagonal & 2 \\
\hline
\end{tabular}

From the three discussing above Table 2 , the evaluation function is

$$
g=q * k * \frac{S}{n}
$$

\subsection{Communication Coordination}

When the effective radius of repeater is larger than or equals to the area radius (some repeater radius can reach more than 100 meters), only a repeater can cover the whole area. In order to mitigate interference, besides the geographical separation, the "continuous tone-coded squelch system" (CTCSS), sometimes nicknamed "private line" (PL), Since a repeater has a special frequency pair and a particular PL, and there are different demand of network serves (different frequencies used for different purposes) and the number of user, the spectrum range need to be divided into multiple channels.

So our task is optimizing frequency channel coordinate and the use of PL according to the users' quantities and purposes on the basis of complete coverage.

\section{$3 \quad$ Results and Analysis}

We already know that the layout scheme of triangular, square and hexagon by model two. The graphics respectively shown as figure...

Hypothesis environmental conditions and sensor performance is the same. Then analyze their advantages and disadvantages below Figure 5.

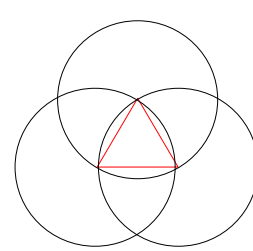

a) triangular

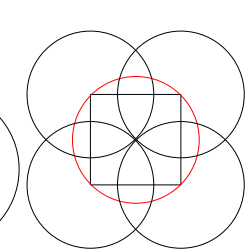

b) square

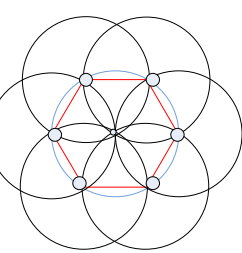

c)hexagon

Fig. 5. Layout scheme 
Use Monte Carlo Simulation to calculate the three area ratio of double covered area with the whole area. The result shows in table 3 and table 4.

Table 3. The area ratio in square

\begin{tabular}{ccc}
\hline Simulation times & The number of nodes & The area ratio \\
\hline 50000 & 268 & 0.00536 \\
80000 & 441 & 0.005513 \\
100000 & 554 & 0.00554 \\
500000 & 2709 & 0.005418 \\
900000 & 4989 & 0.005543 \\
\hline
\end{tabular}

Table 4. The area ratio in hexagon

\begin{tabular}{ccc}
\hline Simulation times & The number of nodes & The area ratio \\
\hline 50000 & 14678 & 0.29356 \\
80000 & 23475 & 0.293438 \\
100000 & 29393 & 0.29393 \\
500000 & 146564 & 0.293128 \\
900000 & 263982 & 0.293313 \\
\hline
\end{tabular}

We have known that the double covered network in triangular scheme is actually covered triple by model two. So the ratio of double covered area with the whole area is 0 that is the positioning accuracy is 1 .

As for square and hexagon, we take the area ratio by most times of simulation to calculate positioning accuracy and function values. The results show in table5:

Table 5. The positioning accuracy and function values

\begin{tabular}{llll}
\hline scheme & triangular & square & hexagon \\
\hline Area ratio & 0 & 0.005543 & 0.293313 \\
positioning accuracy & 1 & 1 & 0.71 \\
function value & $0.866 q^{*} R^{2}$ & $1.333 q * R^{2}$ & $0.922 q * R^{2}$ \\
\hline
\end{tabular}

The higher value of function is, the better scheme of layout is. In order to select better layout scheme, we give a weight to each scheme based on the environment as table 6 shows.

Table 6. The weights based on environment

\begin{tabular}{llll}
\hline environment & Good & Medium & bad \\
\hline triangular & $0--0.3$ & $0.3-0.7$ & $0.7--1$ \\
square & $0.7--1$ & $0.3-0.7$ & $0--0.3$ \\
hexagon & $0--0.3$ & $0.7--1$ & $0.3-0.7$ \\
\hline
\end{tabular}


In conclusion, the layout scheme is as follows.

(1) If the environment condition is good, choose the square scheme.

(2) If the environment condition is medium, choose the hexagon scheme.

(3) If the environment condition is bad, choose the triangular scheme.

From table 3-4, The positioning accuracy of triangular and square scheme can reach to 1 , but is too low in hexagon scheme. So we take the method of reducing the distance of nodes to increase the positioning accuracy.

Use Monte Carlo Simulation; we get the relation of nodes distance and the positioning accuracy. It's show in table7:

Table 7. The relation of nodes distance and the positioning accuracy

\begin{tabular}{cc}
\hline Nodes distance & positioning accuracy \\
\hline$m=R$ & $71 \%$ \\
$m=0.9 R$ & $81 \%$ \\
$m=0.8 R$ & $89.30 \%$ \\
$m=0.7 R$ & $95.90 \%$ \\
$m=0.6 R$ & $99.70 \%$ \\
$m=0.5 R$ & $100 \%$ \\
\hline
\end{tabular}

So the procedure of layout is to choose the scheme based on the environment. And if the hexagon scheme is chosen, choose the nodes distance based on positioning accuracy.

\section{Discussions}

(1) To once complete coverage network, choose the hexagon scheme, and the node lies in the center, the length of sides is the effective radius.

(2) To more than once complete coverage, compared the triangular, square and hexagon scheme, and get a general scheme of multiple complete coverage by calculate the length of sides on the polygon.

(3) To locating network, compared the advantages and disadvantages of triangular, square and hexagon scheme based on positioning accuracy of double complete coverage.

Acknowledgement. This work was supported by 863 programs (2009BADB0B05).

\section{References}

Yuan, R.: Wireless Network Organize Algorithm and the Application in Cotton Warehouse Management, China Agricultural University, Master Dissertation, Beijing (2011)

Yu, H., Li, O., Zhang, X.: Wireless sensor network theory, technology and implementation, Beijing, pp. 100-300 (2008) 
Tran, D.A., Nguyen, T.: Localizatio in Wireless Sensor Networks based on Support Vector Machines

Usman, K.: Localization in Sensor Networks using Message Passing Algorithm

Zhang, H., Hou Jennifer, C.: Maintaining Sensing coverage and connectivity in large sensor networks. Jounral of Ad Hoc and Sensor Wireless Networks 1(1-2), 89-124 (2005)

Yang, B., Yu, H., Li, L., Li, H.: An Energy Efficient Coopenrative Density Control Algorithm in Large Wireless Sensor Networks. In: WCNC 2007 Proceedings (2007)

Klaus, F.: RFID Handbook, 2nd edn. John Wiley \& Sons Ltd, England (2003)

Wang, R.: Treatment of sensor network covering localization method research (2009)

Qi, W.: Wireless sensor network node localization and covering technology research (2008)

Fan, Z.: Wireless sensor network covered with nodes deployment issues research (2008)

Zhang, C.: Hexagon node coverage model research (2010)

Gao, X.: The regional telecommunication covers node localization launch algorithm (2010)

Yang, Z.: Wireless sensor networks positioning problem solving linear programming algorithm (2008)

Yang, C.: Buildings fire monitoring wireless sensor node cover algorithms (2009) 